\title{
Prediction of acid radical ion binding residues by K-nearest neighbors classifier
}

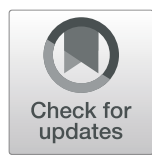

\author{
Liu Liu, Xiuzhen Hu*, Zhenxing Feng, Xiaojin Zhang, Shan Wang, Shuang Xu and Kai Sun
}

From 2018 International Conference on Intelligent Computing (ICIC 2018) and Intelligent Computing and Biomedical Informatics (ICBI) 2018 conference

Wuhan and Shanghai, China. 15-18 August 2018, 3-4 November 2018

\begin{abstract}
Background: Proteins perform their functions by interacting with acid radical ions. Recently, it was a challenging work to precisely predict the binding residues of acid radical ion ligands in the research field of molecular drug design.

Results: In this study, we proposed an improved method to predict the acid radical ion binding residues by using K-nearest Neighbors classifier. Meanwhile, we constructed datasets of four acid radical ion ligand $\left(\mathrm{NO}_{2}{ }^{-}, \mathrm{CO}_{3}{ }^{2-}, \mathrm{SO}_{4}{ }^{2}\right.$ -, $\mathrm{PO}_{4}{ }^{3-}$ ) binding residues from BioLip database. Then, based on the optimal window length for each acid radical ion ligand, we refined composition information and position conservative information and extracted them as feature parameters for K-nearest Neighbors classifier. In the results of 5-fold cross-validation, the Matthew's correlation coefficient was higher than 0.45 , the values of accuracy, sensitivity and specificity were all higher than $69.2 \%$, and the false positive rate was lower than $30.8 \%$. Further, we also performed an independent test to test the practicability of the proposed method. In the obtained results, the sensitivity was higher than $40.9 \%$, the values of accuracy and specificity were higher than $84.2 \%$, the Matthew's correlation coefficient was higher than 0.116 , and the false positive rate was lower than $15.4 \%$. Finally, we identified binding residues of the six metal ion ligands. In the predicted results, the values of accuracy, sensitivity and specificity were all higher than $77.6 \%$, the Matthew's correlation coefficient was higher than 0.6 , and the false positive rate was lower than $19.6 \%$.
\end{abstract}

Conclusions: Taken together, the good results of our prediction method added new insights in the prediction of the binding residues of acid radical ion ligands.

Keywords: K-nearest neighbors classifier, Acid radical ions, Binding residues

\section{Introduction}

The protein is the foundation of life and participates in almost all life processes, such as heredity, growth and development. Most of the proteins need to binding with other specific proteins and form a protein complex to perform their normal biological functions, and previous researchers have made numerous related works and gave us more understanding for the mechanism proteins functions [1-10]. Many proteins require binding to acid radical ions to perform their functions. For instance, protein enzymes bind to phosphate ions $\left(\mathrm{PO}_{4}{ }^{3-}\right)$, which

* Correspondence: hxz@imut.edu.cn

College of Sciences, Inner Mongolia University of Technology, Hohhot 010051, China cause phosphorylation that can regulate enzyme activity [11]; sulfate ion are involved in several important processes of cell metabolism, such as the synthesis process of cysteine and the sulfation process of protein [12, 13]. However, it's still a limit to completely understand the cellular mechanism of protein function. Therefore, it is a valuable work to accurately predict the binding residues of acid radical ion ligands, which can help us illustrate the function of proteins.

Up to now, some researchers have studied acid radical ion binding residues by the experimental methods. In 1966, Pardee used the experimental method to study proteins combining with sulfate ion in Salmonella typhimurium, and analyzed the mechanism of interaction 
between sulfate ion and binding residues [14]. In 2002, the experimental method was adopted by Richard et al. to study the interaction between proteoglycans and sulfate ions, locating the sites of interaction with heparan sulfate in the protein [15]. Tamada studied the sulfation of proteins by the experimental method in 2003 [16]. Some researchers have studied acid radical ion binding residues by the theoretical methods. For instance, $\mathrm{Hu}$ et al. developed the model (IonSeq) for predicting four acid radical ion $\left(\mathrm{NO}_{2}{ }^{-}, \mathrm{CO}_{3}{ }^{2-}, \mathrm{SO}_{4}{ }^{2-}, \mathrm{PO}_{4}{ }^{3-}\right)$ binding residues that were taken from the BioLip database and achieved an accuracy of nearly $98 \%$ for all ions in 2016 [17]. In 2016, $\mathrm{Hu}$ et al. predicted binding residues of $\mathrm{SO}_{4}{ }^{2-}$ and $\mathrm{PO}_{4}{ }^{3-}$ in the BioLip database by the ensemble classifier, and obtained Matthew's correlation coefficient was higher than 0.23 and overall accuracy was higher than $97 \%$ [18]. In 2017, SVM algorithm was used by Li et al. to identify the binding residues of $\mathrm{SO}_{4}{ }^{2-}$ from LPC, achieving the Matthew's correlation coefficient of 0.571 and the overall accuracy of $78.5 \%$ in the five-fold cross-validation [19]. In 2017, Zhang et al. updated the online server COFACTOR by combining structure, sequence and protein-protein interaction information to improve proteins function prediction, in which obtained the Matthew's correlation coefficient was greater than that obtained by Concavity and Findsite for ligandbinding residues of the same set of proteins [20]. 2018, Peyton et al. used an interpretable confidence-rated boosting algorithm to predict protein-ligand interactions with high accuracy from ligand chemical substructures and protein sequence motifs [21].

In this paper, we reconstructed datasets of acid radical ion ligands from BioLip database and developed an improved method to predict the binding residues of four acid radical ions. We explored the optimal window length and extracted the refined characteristics from the composition and position information. Besides, we also integrated the information of amino acid, hydrophilichydrophobic, polarization charge and predicted structure as characteristics parameters for the K-nearest Neighbors classifier. In comparison with previous work, we obtained better results in the predicted of $\mathrm{NO}_{2}{ }^{-}, \mathrm{CO}_{3}{ }^{2-}$ and $\mathrm{PO}_{4}{ }^{3-}$ ligands.

\section{Materials and methods}

\section{Dataset}

BioLip database contains 13 acid radical ion ligands. The proteins interacting with acid radical ions were downloaded from BioLip database and their pairwise sequence identity was below $95 \%$. Then, the proteins with a resolution less than $3 \AA$ and a sequence length above 50 residues were further selected. Finally, the proteins with sequence identity threshold higher than 30\% were removed using the CD-HIT software [22]. Through the above screening, it was found that the number of binding residues of only four acid radical ions $\left(\mathrm{NO}_{2}{ }^{-}, \mathrm{CO}_{3}{ }^{2-}\right.$, $\mathrm{SO}_{4}{ }^{2-}$ and $\mathrm{PO}_{4}{ }^{3-}$ ) conformed to the requirement of statistical analysis. Other acid radical ion ligands, such as $\mathrm{Cl}^{-}, \mathrm{WO}_{4}{ }^{2-}, \mathrm{NO}_{3}{ }^{-}, \mathrm{SO}_{3}{ }^{2-}, \mathrm{PO}_{3}{ }^{3-}$ have fewer number of binding residues. Therefore, we only selected the binding residues of $\mathrm{NO}_{2}{ }^{-}, \mathrm{CO}_{3}{ }^{2-}, \mathrm{SO}_{4}{ }^{2-}$ and $\mathrm{PO}_{4}{ }^{3-}$ as research objects. The non-redundant datasets of the four acid radical ion ligands were shown in Table 1.

Since the interaction between the proteins and ligands is not only related to the binding residues, but surrounding residues also have certain effects, we used the sliding window method to cut the protein sequence into overlapping segments according to the window length of 5 , $7,9,11,13,15$ and 17 amino acid residues, respectively. If the central residue was an acid radical ion binding residue, we grouped it into positive; otherwise, we grouped it into negative segment. In order to ensure that each amino acid residue appeared in the center of the segment, we added a (L-1)/2 dummy residue " $\mathrm{X}$ " at both terminals of the protein chains, where $\mathrm{L}$ is the length of the amino acid sequence segments.

\section{The selection of feature parameters}

Based on our group's previous research on acid radical ion binding residues [19], it was found that the information of amino acid, polarization charge, hydrophilichydrophobic, predicted secondary structures and relative solvent availability could well identify the binding residues of acid radical ion ligands. Therefore, we selected the information of these five basic parameters to predict binding residues of four acid radical ion ligands.

The polarization charge, hydrophilic-hydrophobic, and relative solvent accessibility have different classifications according to various standards. The twenty amino acids are divided into three categories according to the polarization charge, including positively charged amino acids $(K, R, P)$, negatively charged amino acids $(D, E)$, and uncharged amino acids (N, Q, H, L, I, V, A, M, F, S, T, Y, $\mathrm{W}, \mathrm{C}, \mathrm{G})$ [23]; they are grouped into six categories according to the hydrophilic-hydrophobic properties (Table 2) [24]. In this paper, the relative solvent accessibility (RSA) threshold value of $25 \%$ is chosen to indicate whether the residue is exposed (RSA $>25 \%$ ) or buried $(\mathrm{RSA}<25 \%$ ).

There are three predicted secondary structures: $\alpha$-helix $(\mathrm{H}), \beta$-strand $(\mathrm{E})$ and coil $(\mathrm{C})$.

Table 1 Benchmark dataset of four acid radical ions

\begin{tabular}{llll}
\hline Acid radical ion & Chains & Positive segments & Negative segments \\
\hline $\mathrm{NO}_{2}{ }^{-}$ & 22 & 98 & 8144 \\
$\mathrm{CO}_{3}{ }^{2-}$ & 62 & 316 & 22,766 \\
$\mathrm{SO}_{4}{ }^{2-}$ & 303 & 2125 & 99,729 \\
$\mathrm{PO}_{4}{ }^{3-}$ & 339 & 2168 & 112,279 \\
\hline
\end{tabular}


Table 2 Hydrophilic-hydrophobic classification of amino acids

\begin{tabular}{llll}
\hline Classification & Amino Acids & Classification & Amino Acids \\
\hline strongly hydrophobic & $\mathrm{R}, \mathrm{D}, \mathrm{E}, \mathrm{N}, \mathrm{Q}, \mathrm{K}, \mathrm{H}$ & Proline & $\mathrm{P}$ \\
strongly hydrophilic & $\mathrm{L}, \mathrm{I}, \mathrm{V}, \mathrm{A}, \mathrm{M}, \mathrm{F}$ & Glycine & $\mathrm{G}$ \\
weakly hydrophilic & $\mathrm{S}, \mathrm{T}, \mathrm{Y}, \mathrm{W}$ & Cysteine & $\mathrm{C}$ \\
\hline
\end{tabular}

\section{Extraction methods of feature parameters Increment of diversity algorithm}

Increment of diversity (ID) algorithm is of great significance to the research of biology. It has achieved success in the prediction of subcellular localization and protein folds [25, 26]. It not only can be used as an algorithm for prediction, but also can reduce dimension and refine composition information into discrete increment (ID) values. Its use in this paper belonged to the latter. ID algorithm is introduced as follows:

In the state space of dimension $\mathrm{S}$, the measure of diversity for a vector $X:\left[n_{1}, n_{2}, \ldots, n_{s}\right]$ is

$$
D(X)=N \log N-\sum_{i=1}^{s} n_{i} \log n_{i}
$$

In the two state spaces of dimension $\mathrm{S}$, for two vectors $\mathrm{X}:\left[\mathrm{n}_{1}, \mathrm{n}_{2}, \ldots, \mathrm{n}_{\mathrm{s}}\right]$ and $\mathrm{Y}:\left[\mathrm{m}_{1}, \mathrm{~m}_{2}, \ldots, \mathrm{m}_{\mathrm{s}}\right]$, the measure of diversity for mixed diversity resources $\mathrm{X}+\mathrm{Y}$ is

$$
D(X, Y)=(N+M) \log (N+M)-\sum_{i=1}^{s}\left(n_{i}+m_{i}\right) \log \left(n_{i}+m_{i}\right)
$$

Here, $N=\sum_{i=1}^{s} n_{i} \log n_{i}, \mathrm{M}=\sum_{i=1}^{s} m_{i} \log m_{i} . n_{i} / m_{i}$ is the number of occurrences of $i^{t h}$ information symbol in the state space.

The increment of diversity of $\mathrm{X}$ and $\mathrm{Y}$ is

$$
I D(X, Y)=D(X+Y)-D(X)-D(Y)
$$

The measure of diversity is a measure of information diversity, it can describe the uncertainty of the overall information, while the ID is a measure of the spatial similarity between two diversity sources. If $\mathrm{X}$ is similar to $\mathrm{Y}$, the value of $\operatorname{ID}(\mathrm{X}, \mathrm{Y})$ will be small; otherwise, the value of $\mathrm{ID}(\mathrm{X}, \mathrm{Y})$ will be large. For example, amino acid composition information was input to the ID algorithm, two standard discrete sources were constructed by training set. Then we obtained two ID values for each segment of the testing set. Thus, 20-dimensional vector corresponding to frequencies of 20 amino acids of each sequence segment was compressed into two dimensions.
Finally, two ID values were used as feature parameters of the K-nearest Neighbors classifier.

\section{Position weight scoring matrix}

Position weight scoring matrix (PWSM) is a classifier that has achieved great success in the prediction of super-secondary structures and transcription factor binding sites in genomes [27, 28]. The PWSM algorithm was used in this paper to extract feature parameters. The scoring function can be defined as:

$$
S=\frac{\sum_{i=1}^{L} C_{i}\left(m_{i, j}-m_{i, \min }\right)}{\sum_{i=1}^{L} C_{i}\left(m_{i, \max }-m_{i, \min }\right)}
$$

Here,

$$
m_{i, j}=\log \left(\frac{p_{i, j}}{p_{o, j}}\right)
$$

$p_{i, j}=\frac{\left(n_{i, j}+\frac{\sqrt{N_{i}}}{21}\right)}{\left(N_{i}+\sqrt{N_{i}}\right)}$, the conserved parameters of $\mathrm{i}^{\text {th }}$ position is

$$
C_{i}=\frac{100}{\log 21}\left(\sum_{i=1}^{21} p_{i, j} \log p_{i, j}+\log 21\right)
$$

Here $\mathrm{j}$ is 20 amino acids and dummy residue " $\mathrm{X}$ ". $m_{i, j}$ is the matrix element of position weight matrix and denotes the weight probability of the $j^{\text {th }}$ amino acid at the $i^{\text {th }}$ position, $m_{i, \max }$ and $m_{i, \text { min }}$ are the maximum value and minimum value of $m_{i, j}$, respectively. $p_{i, j}$ is the observed probability of the $j^{\text {th }}$ amino acids at the $i^{\text {th }}$ position, and $p_{o, j}$ is the background probability of the $j^{\text {th }}$ amino acid. $n_{i, j}$ is the frequency of $j^{\text {th }}$ amino acids at the $i^{t h}$ position. $N_{i}$ is total number of amino acids at the $i^{t h}$ position. $\mathrm{L}$ is the length of the amino acid sequence segments.

We constructed two standard position weight matrices using binding segments and non-binding segments from training set, respectively. For each segment from the testing set, $2 \mathrm{~L}$-dimensional position information was obtained straightly from standard position weight matrices and two matrix scoring (S) values were obtained by scoring function. Thus, $21^{*} \mathrm{~L}$-dimensional vector corresponding to position conservation of 21 amino acids of each sequence segment was compressed into $2 \mathrm{~L}$ and $2 \mathrm{di}-$ mensions. We used 2-dimensional $\mathrm{S}$ value and $2 \mathrm{~L}$ dimensional position information as feature parameters, respectively. 


\section{Etraction of feature parameters The composition features}

Since the same amino acid residue had different frequencies in the binding segments and the non-binding segments, the amino acid composition information was selected as a feature parameter in this paper. We also selected the composition information of polarization charge, hydrophilic-hydrophobic, secondary structure and relative solvent availability as feature parameters.

\section{The position features}

Since the conservation of amino acids was different at the same position in the binding segments and the nonbinding segments, we selected the $2 \mathrm{~L}$-dimensional position amino acid information as a feature parameter, which was obtained from standard position weight matrices. Similarly, we selected the $2 \mathrm{~L}$-dimensional position information of the polarization charge, hydrophilichydrophobic, secondary structure and relative solvent availability as feature parameters. Then we combined the position information of five basic parameters as feature parameters, namely position combination features and input to the K-nearest Neighbors classifier to identify binding residues of acid radical ion ligands.

\section{The reduced dimension and refined features}

For the amino acid composition information, we obtained the 2-dimensional ID value by using the formula (3). We replaced the amino acid composition with the polarization charge composition, hydrophilichydrophobic composition, secondary structures composition and relative solvent availability composition and obtained the 2-dimensional ID value, respectively. Therefore, we obtained the 10-dimensional ID value.

For the position amino acid information, we obtained the 2-dimensional $\mathrm{S}$ value by using the formula (4). Similarly, for the position information of polarization charge, hydrophilic-hydrophobic, predicted secondary structures and relative solvent availability, we obtained the 2-dimensional $S$ value, respectively. Therefore, we obtained the 10-dimensional $\mathrm{S}$ value.

The 10-dimensional ID value and the 10-dimensional $S$ value were combined as feature parameters, namely the 20-dimensional combination feature and input to the K-nearest Neighbors classifier to predict binding residues of acid radical ion ligands.

\section{K-nearest neighbors classifier}

K-nearest Neighbors (KNN) classifier is a statisticalbased machine learning method, which was proposed by Cover and Hart in 1967 [29]. The basic idea of KNN classifier is that $\mathrm{k}$ nearest samples of a test sample are found by using a distance formula, then the test sample belongs to the category with the largest number in the $\mathrm{k}$ nearest samples. Different $\mathrm{k}$ values will yield different classification results, the performance of $\mathrm{KNN}$ classifier is optimal when $\mathrm{k}$ takes an appropriate value. KNN classifier has been widely used in classification and regression problems, and made a great success in predicting various attributes of proteins, such as proteins subcellular localization and protein structure classification [30, 31].

KNN classifier can get better prediction results when it classifies the dataset with small samples, and the predicted results are more accurate when the number of positive and negative samples of dataset is equal. In this paper, the number of samples used was not large, and negative samples with the equal number of positive samples were randomly sampled. These characteristics matched up the KNN classifier model. Therefore, we used the KNN classifier to identify the four acid radical ion ligand binding residues. Since the algorithm was very mature, we adopted KNN classifier on the weka3.8 platform. The distance formula chosen was Euclidean distance [32-34].

\section{The validation and evaluation metrics}

The proposed method was evaluated by the five-fold cross-validation. The dataset was randomly divided into five equal parts. Four parts were used for training, and remaining one part was used for testing. This process was repeated 5 times, and each part was used once for testing. The average value of five experimental results was taken as the final result. Because the number of negative samples is much larger than that of positive samples, to ensure the stability of the result, negative samples with equal numbers of positive samples were randomly sampled 10 times. The final result was the average value of the 10 results obtained by the five-fold cross-validation.

The following five measures were used to evaluate the prediction performance of acid radical ion binding residues: sensitivity (Sn), specificity (Sp), accuracy (Acc), Matthew's correlation coefficient (MCC) and false positive rate (FPR). These measures were defined as:

$$
\begin{aligned}
& S_{n}=\frac{T P}{T P+F N} \times 100 \% \\
& S_{p}=\frac{T N}{T N+F P} \times 100 \% \\
& A C C=\frac{T P+T N}{T P+T N+F P+F N} \times 100 \% \\
& M C C=\frac{(T P \times T N)-(F P \times F N)}{\sqrt{(T P+F P)(T P+F N)(T N+F P)(T N+F N)}} \\
& F P R=\frac{F P}{T N+F P}
\end{aligned}
$$

Where TP is the number of correctly predicted acid radical ion binding residues, $\mathrm{TN}$ is the number of 


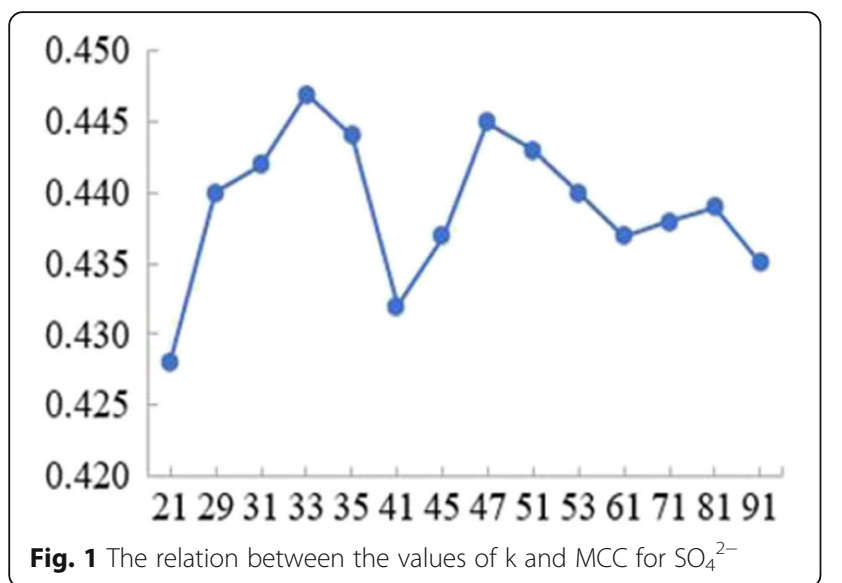

correctly predicted non-binding residues, FP is the number of non-binding residues predicted as binding residues, and FN is the number of binding residues predicted as non-binding residues.

\section{Results and discussion}

The selection of the optimal $k$ value

For each experiment, the best performance obtained by the five-fold cross-validation is achieved with an optimal $k$ value. The average of optimal $k$ values given by 10 experiments is defined as the optimal $\mathrm{k}$ value in the determined window length, and it will be reused in 10 experiments to achieve prediction results. At the window length of 13, taking the selection of optimal $\mathrm{k}$ value of position combination features of $\mathrm{SO}_{4}{ }^{2-}$ ligand as an example, we elaborated on the selection method of the optimal $\mathrm{k}$ value.

Since the negative samples were randomly sampled 10 times, we obtained 10 sample sets. When the window length of $\mathrm{SO}_{4}{ }^{2-}$ ligand was selected 13, we performed the experiments for ten sample sets respectively. Since different k values would obtain different predicted results, for each experiment, the position combination features were input to the KNN

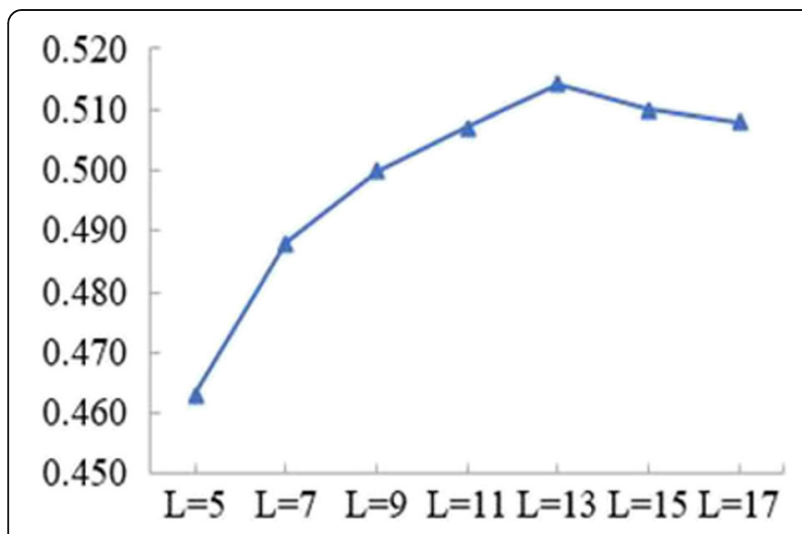

Fig. 2 The MCC values of different $L$
Table 3 Evaluation metrics of position combination features at different $\mathrm{L}$ of $\mathrm{PO}_{4}{ }^{3-}$

\begin{tabular}{lllllll}
\hline $\mathrm{L}$ & Optimal k value & $\mathrm{Sn}(\%)$ & $\mathrm{Sp}(\%)$ & Acc (\%) & FPR (\%) & MCC \\
\hline 5 & 77 & 76.6 & 69.6 & 73.1 & 30.4 & 0.463 \\
7 & 23 & 76.9 & 71.8 & 74.4 & 28.2 & 0.488 \\
9 & 33 & 76.1 & 73.8 & 75.0 & 26.2 & 0.500 \\
11 & 17 & 76.2 & 74.5 & 75.3 & 25.5 & 0.507 \\
13 & 15 & 76.0 & 75.4 & 75.7 & 24.6 & 0.514 \\
15 & 21 & 76.7 & 74.4 & 75.5 & 25.6 & 0.510 \\
17 & 21 & 78.0 & 72.8 & 75.4 & 27.2 & 0.508 \\
\hline
\end{tabular}

classifier to select optimal $k$ value. For one of the experiments, the obtained relation between $\mathrm{k}$ values and the corresponding MCC values was shown in Fig. 1. In Fig. 1, the $\mathrm{x}$-axis represents $\mathrm{k}$ value and the $\mathrm{y}$-axis represents the MCC value. As seen, the highest MCC value was 0.447 and the corresponding $\mathrm{k}$ value was 33. Therefore, the optimal $\mathrm{k}$ value corresponding to the position combination features was 33 in this experiment.

We performed the experiments for other nine sample sets by the same method and the obtained nine optimal $\mathrm{k}$ values were $27,29,31,31,33,35,35,37,39$, respectively. The obtained average value of ten optimal $k$ values was 33. Therefore, at the window length of 13, 33 was the optimal $\mathrm{k}$ value which was selected for the position combination features of $\mathrm{SO}_{4}{ }^{2-}$ ligand.

\section{The selection of the optimal window length}

In the five-fold cross-validation, position combination information was input to the KNN classifier as a characteristic parameter to select the optimal window length $(\mathrm{L})$ of the sequence segments for each acid radical ion. For each window length, based on the position combination features, we selected the optimal $\mathrm{k}$ value for them and input them to the KNN classifier to perform 10 experiments with the optimal $k$ value. The average of results obtained by 10 experiments was the final results at each window length. Therefore, we obtained seven results at window length of 5 , $7,9,11,13,15,17$. The window length corresponding to the highest result of the seven results was the optimal window length. Taking the selection of optimal window length

Table 4 The performance of amino acid composition feature by KNN classifier

\begin{tabular}{lllllll}
\hline Ligand & Optimal k value & $\mathrm{Sn}(\%)$ & $\mathrm{Sp}(\%)$ & Acc (\%) & $\mathrm{FPR}(\%)$ & $\mathrm{MCC}$ \\
\hline $\mathrm{NO}_{2}{ }^{-}$ & 23 & 54.1 & 72.4 & 63.3 & 27.6 & 0.270 \\
$\mathrm{CO}_{3}{ }^{2-}$ & 37 & 63.9 & 52.8 & 58.4 & 47.2 & 0.169 \\
$\mathrm{SO}_{4}{ }^{2-}$ & 91 & 59.3 & 61.1 & 60.2 & 38.9 & 0.204 \\
$\mathrm{PO}_{4}{ }^{3-}$ & 41 & 62.9 & 62.1 & 62.5 & 37.9 & 0.250 \\
\hline
\end{tabular}


Table 5 The performance of composition combination features by KNN classifier

\begin{tabular}{lllllll}
\hline Ligand & Optimal k value & $\mathrm{Sn}(\%)$ & $\mathrm{Sp}(\%)$ & Acc (\%) & FPR (\%) & MCC \\
\hline $\mathrm{NO}_{2}{ }^{-}$ & 77 & 57.1 & 76.5 & 66.8 & 23.5 & 0.343 \\
$\mathrm{CO}_{3}{ }^{2-}$ & 35 & 63.6 & 59.5 & 61.6 & 40.5 & 0.231 \\
$\mathrm{SO}_{4}{ }^{2-}$ & 25 & 66.0 & 61.7 & 63.9 & 38.3 & 0.277 \\
$\mathrm{PO}_{4}{ }^{3-}$ & 71 & 69.1 & 66.3 & 67.7 & 33.7 & 0.355 \\
\hline
\end{tabular}

of $\mathrm{PO}_{4}{ }^{3-}$ ligand as an example, we illustrated the method of the selection of the optimal window length.

For $\mathrm{PO}_{4}{ }^{3-}$ ligand, based on the position combination features, we selected the optimal $k$ value for them at each window length. For each window length, we performed experiments using the optimal $\mathrm{k}$ value for ten sample sets respectively, and obtained final predicted results. The obtained results by the five-fold crossvalidation were given in Fig. 2 and Table 3, respectively.

As shown in Fig. 2, the $\mathrm{MCC}$ value of $\mathrm{PO}_{4}{ }^{3-}$ ligand was increased from window length of 5 to 13. It showed a decreasing tendency from window length of 13 to 17. At window length of $13, \mathrm{PO}_{4}{ }^{3-}$ ligand obtained the highest MCC value. At the same time, the values of Sp, Acc and FPR were the highest and the $S n$ value was approximately equal to that of others when the window length was 13 (see Table 3). Therefore, the optimal window length of $\mathrm{PO}_{4}{ }^{3-}$ ligand was 13 .

The optimal window lengths of the other three acid radical ion ligands were selected by the same way. The selected optimal window lengths of $\mathrm{NO}_{2}{ }^{-}, \mathrm{CO}_{3}{ }^{2-}$ and $\mathrm{SO}_{4}{ }^{2-}$ were 13,15 and 13 , respectively.

\section{Predicted results of composition features}

The amino acid composition information was input to the KNN classifier as a feature parameter to predict binding residues of the four acid radical ion ligands. The predicted results of the five-fold cross-validation were shown in Table 4.

As shown in Table 4, the Acc values were lower than $65 \%$, and the MCC values were lower than 0.3 for four acid radical ion ligands. Especially for $\mathrm{CO}_{3}{ }^{2-}$ ligand, the result was the lowest, with the MCC value of 0.169 and the FPR value of $47.2 \%$. The reason may be that the extracted information was incomplete, so we added the composition information of polarization charge,

Table 6 The performance of position combination features by KNN classifier

\begin{tabular}{lllllll}
\hline Ligand & Optimal k value & $\mathrm{Sn}(\%)$ & $\mathrm{Sp}(\%)$ & $\mathrm{Acc}(\%)$ & $\mathrm{FPR}(\%)$ & $\mathrm{MCC}$ \\
\hline $\mathrm{NO}_{2}{ }^{-}$ & 75 & 81.6 & 61.2 & 71.4 & 38.8 & 0.438 \\
$\mathrm{CO}_{3}{ }^{2-}$ & 31 & 75.6 & 67.7 & 71.7 & 32.3 & 0.435 \\
$\mathrm{SO}_{4}{ }^{2-}$ & 33 & 73.5 & 71.2 & 72.3 & 28.8 & 0.447 \\
$\mathrm{PO}_{4}{ }^{3-}$ & 15 & 76.0 & 75.4 & 75.7 & 24.6 & 0.514 \\
\hline
\end{tabular}

Table 7 Comparison of prediction results of three features

\begin{tabular}{llllllll}
\hline Ligand & Feature & $\begin{array}{l}\text { Optimal } k \\
\text { value }\end{array}$ & $\begin{array}{l}\mathrm{Sn} \\
(\%)\end{array}$ & $\begin{array}{l}\mathrm{Sp} \\
(\%)\end{array}$ & $\begin{array}{l}\text { Acc } \\
(\%)\end{array}$ & $\begin{array}{l}\mathrm{FPR} \\
(\%)\end{array}$ & MCC \\
\hline $\mathrm{NO}_{2}{ }^{-}$ & $\mathrm{C}$ & 77 & 57.1 & 76.5 & 66.8 & 23.5 & 0.343 \\
& $\mathrm{P}$ & 75 & 81.6 & 61.2 & 71.4 & 38.8 & 0.438 \\
& $\mathrm{R}$ & 75 & 81.6 & 79.6 & 80.6 & 20.4 & 0.612 \\
$\mathrm{CO}_{3}{ }^{2-}$ & $\mathrm{C}$ & 35 & 63.6 & 59.5 & 61.6 & 40.5 & 0.231 \\
& $\mathrm{P}$ & 31 & 75.6 & 67.7 & 71.7 & 32.3 & 0.435 \\
& $\mathrm{R}$ & 115 & 74.4 & 78.5 & 76.4 & 21.5 & 0.529 \\
$\mathrm{SO}_{4}{ }^{2-}$ & $\mathrm{C}$ & 25 & 66.0 & 61.7 & 63.9 & 38.3 & 0.277 \\
& $\mathrm{P}$ & 33 & 73.5 & 71.2 & 72.3 & 28.8 & 0.447 \\
& $\mathrm{R}$ & 37 & 75.8 & 69.2 & 72.5 & 30.8 & 0.450 \\
$\mathrm{PO}_{4}{ }^{3-}$ & $\mathrm{C}$ & 71 & 69.1 & 66.3 & 67.7 & 33.7 & 0.355 \\
& $\mathrm{P}$ & 15 & 76.0 & 75.4 & 75.7 & 24.6 & 0.514 \\
& $\mathrm{R}$ & 61 & 76.4 & 74.0 & 75.2 & 26.0 & 0.504 \\
\hline & & & & & & &
\end{tabular}

hydrophilic-hydrophobic, secondary structure and relative solvent accessibility for further prediction. The prediction results of the five-fold cross-validation were shown in Table 5. As seen, the performance was improved after adding other composition features. For example, the MCC value was significantly improved from 0.250 to 0.355 , the $\mathrm{Sn}$ value was increased from 62.9 to $69.1 \%$, and the Acc value was increased by $5.2 \%$ for $\mathrm{PO}_{4}{ }^{3-}$ ligand. It indicates that the newly added features contained valid information, which has great significance for identifying acid radical ion binding residues.

\section{Predicted results of position combination features}

Since the predicted results of the composition features were not good enough, position combination information of amino acid, polarization charge, hydrophilichydrophobic, secondary structure and relative solvent accessibility were used as characteristic parameters to recognize four acid radical ion binding residues by KNN classifier. Obtained results of the five-fold crossvalidation were shown in Table 6.

As seen, the result of $\mathrm{PO}_{4}{ }^{3-}$ ligand was the highest, the MCC value was 0.514 , and the values of Acc, Sn and Sp were all higher than $75 \% . \mathrm{PO}_{4}{ }^{3-}$ ligand was sensitive

Table 8 The data of the training dataset and independent test dataset

\begin{tabular}{lllllllll}
\hline Ligand & \multicolumn{3}{l}{ Training dataset } & & \multicolumn{3}{c}{ Independent test dataset } \\
\cline { 2 - 3 } & Chains & $\mathrm{P}^{\mathrm{a}}$ & $\mathrm{N}^{\mathrm{b}}$ & & Chains & $\mathrm{P}^{\mathrm{a}}$ & $\mathrm{N}^{\mathrm{b}}$ \\
\hline $\mathrm{NO}_{2}{ }^{-}$ & 17 & 76 & 6218 & & 5 & 22 & 1926 \\
$\mathrm{CO}_{3}{ }^{2-}$ & 49 & 252 & 18,066 & & 13 & 64 & 4700 \\
$\mathrm{SO}_{4}{ }^{2-}$ & 242 & 1751 & 79,164 & 61 & 374 & 20,565 \\
$\mathrm{PO}_{4}{ }^{3-}$ & 271 & 1730 & 90,786 & 68 & 438 & 21,493 \\
\hline
\end{tabular}

${ }^{a}$ The number of positive (binding) samples

${ }^{\mathrm{b}}$ The number of negative (non-binding) samples 
Table 10 Benchmark dataset of six metal ion ligands

\begin{tabular}{llll}
\hline Metal ions & Chains & Binding residues & Non-binding residues \\
\hline $\mathrm{Zn}^{2+}$ & 1428 & 6408 & 405,113 \\
$\mathrm{Fe}^{2+}$ & 92 & 382 & 29,345 \\
$\mathrm{Fe}^{3+}$ & 217 & 1057 & 68,829 \\
$\mathrm{Cu}^{2+}$ & 117 & 485 & 33,948 \\
$\mathrm{Mn}^{2+}$ & 459 & 2124 & 156,625 \\
$\mathrm{Co}^{2+}$ & 194 & 875 & 55,050 \\
\hline
\end{tabular}

to the position combination features and could be well identified by position features. However, the predicted results of $\mathrm{NO}_{2}{ }^{-}, \mathrm{CO}_{3}{ }^{2-}$ and $\mathrm{SO}_{4}{ }^{2-}$ were less accurate, in which the MCC values were lower than 0.5 and the Acc values were lower than $73 \%$. Probably because these acid radical ion binding residues were less sensitive to position information.

\section{Predicted results of reduced dimension and refined features}

Since predicted results of binding residues of $\mathrm{NO}_{2}{ }^{-}$, $\mathrm{CO}_{3}{ }^{2-}$ and $\mathrm{SO}_{4}{ }^{2-}$ were still lower, the 20-dimensional combination feature was input to the KNN classifier to predict binding residues of acid radical ion ligands. The predicted results of the five-fold cross-validation were given in Table 7.

As seen, the 20-dimensional combination feature obtained the better results. The values of Acc and Sn were higher than $70 \%$, and the MCC values were higher than 0.45 for four acid radical ions. There are two possible reasons for it. One is that there is complementarity between the composition information and the position information. The other is that the 20-dimensional combination feature does not have redundant information.

\section{The comparison of predicted results among three kinds of features}

To make the comparison of predicted results among composition combination features $(\mathrm{C})$, position combination features (P) and 20-dimensional combination feature (R) more obvious, their results of the five-fold cross-validation obtained by KNN classifier were listed in Table 7.

Compared with the results obtained by composition combination features, the values of MCC, Acc and Sp were increased by using position combination features for all acid radical ions. For example, the MCC value of $\mathrm{CO}_{3}{ }^{2-}$ ligand was apparently increased from 0.231 to 0.435 , the $\mathrm{Sn}$ value of $\mathrm{NO}_{2}{ }^{-}$ligand was obviously increased from 57.1 to $81.6 \%$. It shows that position combination features contain more valid information.

Compared with the results obtained by position combination features, the predicted results of the 20dimensional combination feature were better. The predicted results of $\mathrm{NO}_{2}{ }^{-}$and $\mathrm{CO}_{3}{ }^{2-}$ ligands were significantly improved. In terms of $\mathrm{NO}_{2}{ }^{-}$ligand, the MCC value was obviously improved from 0.438 to 0.612 , the Acc value was increased from 71.4 to $80.6 \%$, and the values of $\mathrm{Sn}$ and $\mathrm{Sp}$ were balanced between 81.6 and $79.6 \%$, respectively. For $\mathrm{CO}_{3}{ }^{2-}$ ligand, the FPR value was decreased from 32.3 to $21.5 \%$, the values of Acc and MCC were increased by 4.7 and $9.4 \%$, respectively. As for the $\mathrm{SO}_{4}{ }^{2-}$ ligand, the $\mathrm{MCC}$ value was improved from 0.447 to 0.450 with almost no change. For $\mathrm{PO}_{4}{ }^{3-}$ ligand, although the values of Acc, MCC and Sp were slightly declined, the Sn value was slightly increased. It may be that 20-dimensional combination feature lost some valid information, resulting in decrease of negative sample identification results.

In the above predicted results, the identification results corresponding to the 20-dimensional combination feature were the best. Therefore, we should select the feature parameters which contain more valid information and input them to the KNN classifier to accurately recognize binding residues of acid radical ion ligands.

\section{Predicted results of independent test}

To evaluate the practicability of KNN classifier, we have made the independent test for four acid radical ion ligand binding residues.

Table 9 Comparison of our independent test with lonSeq

\begin{tabular}{lllllllll}
\hline Ligand & Method & $\mathrm{L}$ & Optimal k value & Sn (\%) & Sp (\%) & Acc (\%) & FPR(\%) & MCC \\
\hline $\mathrm{NO}_{2}{ }^{-}$ & IonSeq & 11 & - & 18.00 & 99.78 & 98.79 & - & 0.2847 \\
& OUR'S & 13 & 75 & 40.90 & 98.60 & 97.90 & 1.40 & 0.3100 \\
$\mathrm{CO}_{3}{ }^{2-}$ & IonSeq & 13 & - & 10.62 & 99.82 & 98.58 & - & 0.2127 \\
& OUR'S & 15 & 115 & 48.40 & 95.00 & 94.40 & 5.00 & 0.2170 \\
$\mathrm{SO}_{4}{ }^{2-}$ & IonSeq & 11 & - & 13.65 & 99.32 & 97.53 & - & 0.1906 \\
& OUR'S & 13 & 37 & 43.90 & 86.80 & 85.80 & 13.20 & 0.1160 \\
$\mathrm{PO}_{4}{ }^{3-}$ & IonSeq & 11 & - & 24.15 & 99.38 & 97.95 & - & 0.3121 \\
& OUR'S & 13 & 61 & 63.20 & 84.60 & 84.20 & 15.40 & 0.1810 \\
\hline
\end{tabular}


Table 11 Comparison of results between KNN classifier with SVM

\begin{tabular}{|c|c|c|c|c|c|c|c|c|}
\hline Ligand & Method & L & Optimal k value & Sn (\%) & Sp (\%) & Acc (\%) & FPR (\%) & MCC \\
\hline \multirow[t]{2}{*}{$\overline{Z n^{2+}}$} & OUR'S & 7 & 103 & 94.3 & 83.8 & 89.1 & 16.2 & 0.786 \\
\hline & SVM & & - & 99.8 & 99.5 & 99.7 & - & 0.993 \\
\hline \multirow[t]{2}{*}{$\mathrm{Fe}^{2+}$} & OUR'S & 9 & 41 & 92.1 & 80.4 & 86.3 & 19.6 & 0.730 \\
\hline & SVM & & - & 91.9 & 90.7 & 91.3 & - & 0.826 \\
\hline \multirow[t]{2}{*}{$\mathrm{Fe}^{3+}$} & OUR'S & 9 & 15 & 84.6 & 84.9 & 84.7 & 15.1 & 0.694 \\
\hline & SVM & & - & 86.9 & 88.7 & 87.8 & - & 0.756 \\
\hline \multirow[t]{2}{*}{$\mathrm{Cu}^{2+}$} & OUR'S & 13 & 49 & 92.4 & 86.6 & 89.5 & 13.4 & 0.791 \\
\hline & SVM & & - & 95.5 & 97.1 & 96.3 & - & 0.926 \\
\hline \multirow[t]{2}{*}{$\mathrm{Mn}^{2+}$} & OUR'S & 7 & 23 & 79.1 & 80.9 & 80.0 & 19.1 & 0.600 \\
\hline & SVM & & - & 82.1 & 84.4 & 83.2 & - & 0.664 \\
\hline \multirow[t]{2}{*}{$\mathrm{CO}^{2+}$} & OUR'S & 11 & 99 & 77.6 & 83.1 & 80.3 & 16.9 & 0.608 \\
\hline & SVM & & - & 80.8 & 85.1 & 83.0 & - & 0.660 \\
\hline
\end{tabular}

The dataset of four acid radical ion binding residues was divided into two parts, including training dataset that was used to train model and the independent test dataset that was used to test model. The protein chains in training dataset accounted for $80 \%$ of the total data. The data of the two datasets was shown in Table 8 .

In the independent test, we used the optimal window length of each acid radical ion was taken from Section 3.2. Since acid radical ion ligands were sensitive to the 20dimensional combination feature, we extracted it as feature parameter of independent testing and input it to the KNN classifier to identify binding residues of acid radical ion ligands, in which selected optimal $\mathrm{k}$ values were same as Section 3.5. The obtained results were given in Table 9. Besides, the obtained results were compared with that by IonSeq. The results of IonSeq method were taken from literature [17] in which it was obtained by cross-validation.

As seen from Table 9, the Sn values obtained by KNN classifier were all higher than those obtained by IonSeq for all acid radical ions. The MCC values of $\mathrm{NO}_{2}{ }^{-}$and $\mathrm{CO}_{3}{ }^{2-}$ ligands by $\mathrm{KNN}$ classifier were slightly higher than that by IonSeq, while the $\mathrm{MCC}$ values of $\mathrm{SO}_{4}{ }^{2-}$ and $\mathrm{PO}_{4}{ }^{3-}$ ligands were lower than that by IonSeq. There are three possible reasons for it. First, the datasets used are different for two models. IonSeq model is aimed at imbalanced dataset, while model constructed in this paper is aimed at dataset with equal number of positive and negative samples. Second, the model in this paper is tested by independent test, but the IonSeq model is tested by cross-validation. Third, the feature parameters used are different. Both methods have their own advantages and can only be roughly compared.

\section{Predicted results of metal ion ligand binding residues}

In order to test the reliability of proposed method, we used the KNN classifier to predict binding residues of the six kinds of metal ion $\left(\mathrm{Zn}^{2+}, \mathrm{Fe}^{2+}, \mathrm{Fe}^{3+}, \mathrm{Cu}^{2+}, \mathrm{Mn}^{2+}\right.$,
$\left.\mathrm{Co}^{2+}\right)$ with more binding proteins, and compared the results with that obtained by SVM used in literature [35]. Dataset of six kinds of metal ion binding residues used in this paper was taken directly from literature [35] (Table 10). Since the predicted results of acid radical ion binding residues were higher at the 20-dimensional combination feature, it was also extracted as feature parameter and input to the KNN classifier to predict binding residues of six metal ions. The obtained results of the five-fold cross-validation were given in Table 11.

Table 11 shows the prediction results of 6 kinds of metal ion ligand binding residues obtained by KNN classifier. The MCC values were higher than 0.6 , the values of Acc and Sp were higher than $80 \%$, and FPR percentages were lower than $20 \%$. Predicted results of $\mathrm{Zn}^{2+}$ and $\mathrm{Cu}^{2+}$ by KNN classifier were relatively better.

Although the predicted results of six metal ions obtained by KNN classifier were lower than that by SVM, their predicted trends were consistent [35]. To achieve better results, the KNN classifier only need to select an optimal k value, while the SVM need to select a group (c, g) optimal values. KNN classifier can achieve similar prediction results with SVM by simpler calculation. The training time complexity of KNN classifier is lower than that of SVM algorithm. The KNN classifier mainly relies on the surrounding limited adjacent samples rather than the method of discriminating class domain to determine the category, and new data can be added directly to the dataset without retraining. KNN classifier theory is simple and easy to implement. Therefore, KNN classifier can be used as auxiliary tool for predicting acid radical ion ligand binding residues.

\section{Conclusion}

To perform the normal biological functions, many proteins require bind to the specific acid radical ions [1113]. In order to illustrate the proteins function, it is a valuable work to predict the binding residues of acid 
radical ion ligands in recent years. In this work, we proposed K-nearest Neighbors classifier to predict four acid radical ion ligands binding residues. Firstly, the dataset of acid radical ion ligands was constructed. Then we selected the optimal window length for each acid radical ion ligand. Next, we extracted the composition features, position features and reduced dimension and refined features at the optimal window length, and selected the optimal $\mathrm{k}$ value for three feature parameters. The promising results were obtained by K-nearest Neighbors classifier when feature parameters that contained more comprehensive information were used to predict acid radical ion binding residues. In the predicted results, $\mathrm{NO}_{2}{ }^{-}, \mathrm{CO}_{3}{ }^{2-}$ and $\mathrm{PO}_{4}{ }^{3-}$ ligands obtained better results by K-nearest Neighbors classifier. For $\mathrm{SO}_{4}{ }^{2-}$ ligand, other valid information needed to be added in our further work to improve the recognition result.

\section{Abbreviations}

Acc: Accuracy; FPR: False positive rate; ID: Increment of diversity; KNN: Knearest Neighbors; LPC: Ligand Protein Contact; MCC: Matthew's correlation coefficient; PWSF: Position weight scoring matrix; RSA: Relative solvent accessibility; Sn: Sensitivity; Sp: Specificity; SVM: Support vector machine

\section{Acknowledgements}

We are grateful to Deshuang Huang for her guidance on research work and Qiwen Dong's guidance on translation of the paper.

\section{About this supplement}

This article has been published as part of BMC Molecular and Cell Biology Volume 20 Supplement 3, 2019: Proceedings of the 2018 International

Conference on Intelligent Computing (ICIC 2018) and Intelligent Computing and Biomedical Informatics (ICBI) 2018 conference: molecular and cell biology. The full contents of the supplement are available online at https:// bmcmolcellbiol.biomedcentral.com/articles/supplements/volume-20supplement-3.

\section{Authors' contributions}

LL performed the experiments and wrote the paper. XH designed the experiments and analyzed the results. ZF, XZ, SW, SX and KS gave guidance on the writing of the paper. All authors read and approved the final manuscript.

\section{Funding}

Publication costs are funded by National Natural Science Foundation of China (61961032) and Natural Science Foundation of the Inner Mongolia of China (2019BS03025).

\section{Availability of data and materials}

The datasets used and analysed during the current study are available from the corresponding author on reasonable request.

\section{Ethics approval and consent to participate}

Not applicable.

\section{Consent for publication}

Not applicable.

\section{Competing interests}

The authors declare that they have no competing interests.

Published: 11 December 2019

\section{References}

1. Zhu L, Deng SP, et al. Identifying spurious interactions in the proteinprotein interaction networks using local similarity preserving embedding [J]. IEEE/ACM Trans Comput Biol Bioinform. 2017;14(2):345-52.
2. Deng SP, Huang DS. SFAPS: an R package for structure/function analysis of protein sequences based on informational spectrum method []]. Methods. 2014;69(3):207-12

3. Huang DS, Zhang $L$, et al. Prediction of protein-protein interactions based on protein-protein correlation using least squares regression. Curr Protein Pept Sci. 2014;15(6):553-60.

4. Huang DS, Yu HJ. Normalized feature vectors: a novel alignment-free sequence comparison method based on the numbers of adjacent amino acids [J]. IEEE/ACM Trans Comput Biol Bioinform. 2013;10(2):457-67.

5. You ZH, Lei YK, Gui J, et al. Using manifold embedding for assessing and predicting protein interactions from high-throughput experimental data [J]. Bioinformatics. 2010;26(21):2744-51.

6. Xia JF, Zhao XM, Song J, et al. APIS: accurate prediction of hot spots in protein interfaces by combining protrusion index with solvent accessibility [J]. Bmc Bioinformatics. 2010;11(1):174.

7. Xia JF, Zhao XM, et al. Predicting protein-protein interactions from protein sequences using meta predictor []]. Amino Acids. 2010;39(5):1595-9.

8. Xia JF. Kyungsook Han, et al. sequence-based prediction of protein-protein interactions by means of rotation forest and autocorrelation descriptor [J]. Protein Pept Lett. 2010;17(1):137-45.

9. Shi MG, Xia JF, et al. Predicting protein-protein interactions from sequence using correlation coefficient and high-quality interaction dataset [J]. Amino Acids. 2010;38(3):891-9.

10. Wang B. Hau san Wong, et al. inferring protein-protein interacting sites using residue conservation and evolutionary information [J]. Protein Pept Lett. 2006;13(10):999-1005.

11. Burnett G. Kennedy E P, the enzymatic phosphorylation of proteins [J]. Biol Chem. 1954;211:969-80.

12. Thomas L, Michael M. Cloning of a cDNA encoding ATP sulfurylase form Arabidopsis thaliana by functional expression in Saccharomyces cerevisiae [J]. Plant Physiol. 1994;105:897-902.

13. Monigatti F, Gasteiger E, Bairoch A, et al. The sulfinator: predicting tyrosine sulfation sites in protein sequences [J]. Bioinformatics. 2002;18:769-70.

14. Pardee B. Purification and properties of a sulfate-bindind protein form Salmonella typhimurium [J]. Received for publication. J Biol Chem. 1966;24:5886-92.

15. Richard G. Christoph Hundt, Stefan Weiss, et al. Turnbull. Identification of the Heparan sulfate binding sites in the cellular prion protein [J]. J Biol Chem. 2002:277:18421-30.

16. Tamada Y. Sulfation of silk fibroin by sulfuric acid and anticoagulant activity [J]. Appl Polym Sci. 2003;87:2377-82.

17. Hu X, Dong Q, Yang J, et al. Recognizing metal and acid radical ion-binding sites by integrating ab initio modeling with template-based transferals []]. Bioinformatics. 2016;32(23):btw396.

18. Hu X, Wang K, Dong Q. Protein ligand-specific binding residue predictions by an ensemble classifier [J]. BMC Bioinformatics. 2016;17(1):470.

19. Li S, Hu X, et al. Identifying the sulfate ion binding residues in proteins [J]. International Conference on Biomedical and Biological Engineering, 2017.

20. Zhang C, Freddolino P L, Zhang Y. COFACTOR: improved protein function prediction by combining structure, sequence and protein-protein interaction information[J]. Nucleic Acids Res. 2017;45:W295.

21. Greenside P, Hillenmeyer M, Kundaje A. Prediction of protein-ligand interactions from paired protein sequence motifs and ligand substructures [C]. Pacific Symposium, 2018.

22. Li W, Godzik A. Cd-hit: a fast program for clustering and comparing large sets of protein or nucleotide sequences [M]. Bioinformatics. 2006;22:1658-9.

23. Taylor WR. The classification of amino acid conservation [J]. J Theor Biol. 1986;1 19(2):205-18.

24. Pánek J, Eidhammer I, Aasland R. A new method for identification of protein (sub) families in a set of proteins based on hydropathy distribution in proteins [J]. Proteins Struct Funct Bioinformatics. 2005;58(4):923-34.

25. Feng Z, Hu X. Recognition of 27-class protein folds by adding the interaction of segments and motif information. Biomed Res Int. 2014; 2014(4):871-82.

26. Chen YL, Li QZ. Prediction of the subcellular location of apoptosis proteins. J Theor Biol. 2007;245(4):775-83.

27. Gao S, Hu X. Prediction of four kinds of super secondary structure in enzymes by using ensemble classifier based on scoring SVM [J]. Hans J Comput Biol. 2014;04(1):1-11.

28. Kel AE, GoBling E, Reuter I, el al. MATCHTM: a tool for searching transcription factor binding sites in DNA sequences[J]. Nucleic Acids Res. 2003;13:3576-9. 
29. Thomas Cover PH. Nearest nei^Bor pattern classification []]. IEEE T Inform Theory. 1967;13:21

30. Qian S, Yan B, Li J. Ensemble learning for protein multiplex subcellular localization prediction based on weighted KNN with different features [J]. Applined Intell. 2017;1:1-12.

31. Zhang T, Ding Y, Chou K. Prediction protein structural classes with pseudoamino acid composition: approximate entropy and hydrophobicity pattern [J]. J Theor Biol. 2008;250(1):186-93.

32. Feng ZX, Li QZ. Recognition of long-range enhancer-promoter interactions by adding genomic signatures of segmented regulatory regions [J]. Genomics. 2017:109(5-6):341.

33. Hall M, Frank E, Holmers G, Pfahringer B, Reotemann P, Witten IH. The WEKA software an update. ACM SIGKDD Explor Newsl. 2019;11:10-8.

34. AHa D, Kibler D. Instance-based learning algorithms. Mach Learn. 1991;6:37-66.

35. Cao $X$, Hu X, Zhang $X$, et al. Identification of metal ion binding sites based on amino acid sequences [J]. PLoS One. 2017;12(8):13.

\section{Publisher's Note}

Springer Nature remains neutral with regard to jurisdictional claims in published maps and institutional affiliations.

Ready to submit your research? Choose BMC and benefit from:

- fast, convenient online submission

- thorough peer review by experienced researchers in your field

- rapid publication on acceptance

- support for research data, including large and complex data types

- gold Open Access which fosters wider collaboration and increased citations

- maximum visibility for your research: over $100 \mathrm{M}$ website views per year

At $\mathrm{BMC}$, research is always in progress.

Learn more biomedcentral.com/submissions 